\title{
Duals of vector-valued Köthe function spaces
}

\author{
By MIGUEL FLORENCIO, PEDRO J. PAÚL AND CARMEN SÁEZ \\ E.S. Ingenieros Industriales, Avda, Reina Mercedes s/n, 41012 Sevilla, Spain
}

(Received 1 August 1991 ; revised 24 October 1991)

\begin{abstract}
Let $\Lambda$ be a perfect Köthe function space in the sense of Dieudonné, and $\Lambda^{\times}$its Köthe-dual. Let $E$ be a normed space. Then the topological dual of the space $\Lambda(E)$ of $\Lambda$-Bochner integrable functions equals the corresponding $\Lambda^{\times}\left(E^{\prime}\right)$ if and only if $E^{\prime}$ has the Radon-Nikodým property. We also give some results concerning barrelledness for spaces of this kind.
\end{abstract}

\section{Introduction}

The purpose of this paper is to study several results concerning vector function spaces with values in a normed space. These spaces are obtained as a generalization of the Köthe function spaces studied by Dieudonné[3].

Let $\Lambda$ be a Köthe space defined on a measure space $X$ and $\Lambda^{\times}$its Köthe-dual, and let $E$ be a normed space with dual $E^{\prime}$. We define the space $\Lambda(E)$ of $\Lambda$-Bochner integrable functions as the space of (classes of) strongly measurable functions $f: X \rightarrow E$ such that the composition $\|f(\cdot)\|$ is a function in $\Lambda$. There is a natural topology on $\Lambda(E)$ defined from the topology of $\Lambda$ and the norm on $E$, and the locally convex structure of these spaces has been studied by Clauzure[1], Jordán Lluch and Torregrosa Sánchez [6], Macdonald [8], and Phuong-Các[9], among others.

Macdonald [8] provides a characterization of the dual of $\Lambda(E)$ when $E$ is a locally convex Hausdorff space and the topology on $\Lambda$ is compatible with the dual pair $\left(\Lambda, \Lambda^{\times}\right)$. This characterization is not easy to use even when $E$ is a normed space, in which case it reads as follows : the topological dual of $\Lambda(E)$ can be identified with the set of all $\sigma\left(E^{\prime}, E\right)$-measurable functions $g: X \rightarrow E^{\prime}$ satisfying that for every compact set $\mathbf{K} \subset X$, the restriction $\left.g\right|_{K}$ can be written as $\left.g\right|_{K}=\phi \cdot h$ where $h$ is a $\sigma\left(E^{\prime}, E\right)$ measurable function such that $\|h(\cdot)\| \leqslant 1$ almost everywhere and $\phi$ is in $\Lambda^{\times}$.

In some particular cases, e.g. when $E$ is a reflexive Banach space or $E$ is a Banach space with separable dual, the characterization above can be considerably simplified, the result reducing to $\Lambda(E)^{\prime}=\Lambda^{\times}\left(E^{\prime}\right)$. This situation is much more manageable and comparable with the case of vector-valued sequence spaces studied by Rosier [10]. It seems, then, desirable to characterize those normed spaces $E$ for which the dual of $\Lambda(E)$ equals $\Lambda^{\times}\left(E^{\prime}\right)$. For the case of Bochner integrable functions it is well-known that the characterization of the dual of $L^{1}(E)$ as $L^{\infty}\left(E^{\prime}\right)$ is equivalent to $E^{\prime}$ having the Radon-Nikodým property ([2], IV·1, theorem 1, p. 98). This is also the case when $\Lambda$ is a Fréchet echelon space and $E$ is a Banach space [6]. Here we prove that this characterization holds in general. In the last section, we study the permanence 
of some properties related to barrelledness. Concerning the references given above, we think that this paper can be considered as a continuation of N. Phuong-Các's paper [9].

\section{Terminology and notation}

We refer the reader to Köthe's monograph [7] and to the book [2] of Diestel and Uhl for the terminology used in this paper.

Let $X$ be a locally compact and Hausdorff topological space and $\mu$ be a nonnegative Radon measure on $X$. We assume that $X$ is countable at infinity, so that we can write $X=\bigcup_{n=1}^{\infty} X_{n}$, where each $X_{n}$ is compact and $X_{n} \subset \operatorname{int}\left(X_{n+1}\right)$. We shall always identify those functions that are equal almost everywhere with respect to $\mu$ ( $\mu$-a.e. in short).

We denote by $\Omega$ the space of all locally integrable real-valued functions defined on $X$, and by $\Phi$ the subspace of all measurable essentially bounded functions with compact support. When $X$ is compact we obtain the spaces $L^{1}(\mu)$ and $L^{\infty}(\mu)$ respectively.

For a subset $\Lambda \subset \Omega$ its Köthe-dual is the set $\Lambda^{\times}$of all $f \in \Omega$ such that $f \cdot g$ is integrable for each $g \in \Lambda$. We have $\Lambda \subset \Lambda^{\times \times}=\left(\Lambda^{\times}\right)^{\times}$, and $\Lambda$ is said to be a perfect space if $\Lambda=\Lambda^{\times \times}$. When $\Lambda$ is a subspace of $\Omega$ containing $\Phi$, in particular if $\Lambda$ is perfect, then $\Lambda$ and $\Lambda^{\times}$are put into duality by the canonical bilinear form on $\Lambda \times \Lambda^{\times}$:

$$
(f, g) \mapsto\langle f, g\rangle=\int_{X} f(t) \cdot g(t) d \mu(t)
$$

A subset $H$ of $\Omega$ is said to be normal (or solid) if whenever $H$ contains $f$ it also contains all $g \in \Omega$ satisfying $|g(t)| \leqslant|f(t)| \mu$-a.e. The strong topology $\beta\left(\Lambda, \Lambda^{\times}\right)$on $\Lambda$ equals the polar topology on $\Lambda$ generated by the family $\mathscr{B}$ of all normal and $\sigma\left(\Lambda^{\times}, \Lambda\right)$-bounded subsets of $\Lambda^{\times}$, and it is given by the seminorms

$$
p_{M}: f \in \Lambda \mapsto p_{M}(f):=\sup \left\{\int_{X}|f(t) . g(t)| d \mu(t): g \in M\right\} \quad(M \in \mathscr{B}) .
$$

In what follows, $\Lambda$ remains a perfect Köthe space endowed with its strong topology $\beta\left(\Lambda, \Lambda^{\times}\right)$. We refer the reader to Dieudonné's paper [3] for additional information about Köthe function spaces.

Let $E$ be a normed space with dual $E^{\prime}$. We define the space $\Lambda(E)$ of $\Lambda$-Bochner integrable functions as the space of (classes of) strongly measurable functions $f: X \rightarrow E$ such that the composition $\|f(\cdot)\|$ is a function in $\Lambda$.

Starting from the strong topology $\beta\left(\Lambda, \Lambda^{\times}\right)$on $\Lambda$ and the norm on $E$, we can generate in $\Lambda(E)$ a natural topology defined by the seminorms

$$
q_{M}(f):=p_{M}(\|f(\cdot)\|)=\sup \left\{\int_{X}\|f(t)\| \cdot|g(t)| d \mu(t): g \in M\right\} \quad(M \in \mathscr{B}) .
$$

We will refer to this topology as the natural topology of $\Lambda(E)$. When $\Lambda=L^{p}(\mu)$ with $1 \leqslant p \leqslant+\infty$, we obtain the space $L^{p}(\mu, E)$ of $p$-Bochner integrable functions (see [2], IV 1, p. 97). In particular, $L^{\infty}(\mu, E)$ is the space of strongly measurable, essentially bounded functions from $X$ into $E$ with the ess-sup norm. Denote by $B$ the unit ball of $L^{\infty}(\mu, E)$; thus a function $h: X \rightarrow E$ is in $B$ if it is $\mu$-measurable 
and $\|h(t)\| \leqslant 1 \mu$-a.e. Note that if $\phi \in \Lambda$ and $h \in B$ then the function $f$ given by $f(t):=\phi(t) . h(t)$ is in $\Lambda(E)$. On the other hand, every function $f \in \Lambda(E)$ can be decomposed in the same way, namely $\phi(t)=\|f(t)\|$ and

$$
h(t)= \begin{cases}f(t) \cdot\|f(t)\|^{-1} & \text { if }\|f(t)\| \neq 0 \\ 0 & \text { otherwise. }\end{cases}
$$

Using this decomposition and the form of the seminorms $q_{M}$, it is easy to check that the bounded sets of $\Lambda(E)$ are obtained from the bounded sets of $\Lambda$ by multiplication with $B$, i.e. a set $S \subset \Lambda(E)$ is bounded if and only if there is a bounded set $R \subset \Lambda$ such that $S \subset R . B$.

Two properties of these spaces that we shall use are the fact that the space of compact support simple functions is dense on $\Lambda(E)$ for the natural topology ([9], proposition 1), and that if $E$ is a Banach space, then $\Lambda(E)$ is complete ([9], proposition 2).

\section{A characterization of the dual of vector-valued Köthe function spaces}

We say that a Banach space $E$ has the Radon-Nikodým property with respect to $\mu$ if whenever $G: X \rightarrow E$ is a $\sigma$-additive vector measure that has bounded variation and vanishes on sets of measure zero, there exists a Bochner integrable function $g \in L^{1}(\mu, E)$ such that

$$
G(A)=\int_{A} g d \mu .
$$

In the sequel, we will use the following lemma which was stated in [4].

Lemma. Let $g: X \rightarrow E^{\prime}$ and $\epsilon: X \rightarrow \mathbb{R}$ be $\mu$-measurable functions, $\epsilon$ in addition strictly positive. Then there exists a $\mu$-measurable function $y: X \rightarrow E$ such that

(1) $y$ is countably valued with values in the unit ball of $E$, and

(2) $\|g(t)\| \leqslant\langle g(t), y(t)\rangle+\epsilon(t) \mu$-a.e. in $X$.

Theorem 1. Assume that the dual of $\Lambda\left(\beta\left(\Lambda, \Lambda^{\times}\right)\right)$is $\Lambda^{\times}$. Then $\Lambda(E)^{\prime}=\Lambda^{\times}\left(E^{\prime}\right)$ if and only if $E^{\prime}$ has the Radon-Nikodym property with respect to $\mu$.

Proof. We start by noting that $\Lambda^{\times}\left(E^{\prime}\right)$ can always be identified with a subspace of $\Lambda(E)^{\prime}$. Indeed, for $g \in \Lambda^{\times}\left(E^{\prime}\right)$ consider the element

of $\Lambda(E)^{\prime}$. We have

$$
f \mapsto\langle g, f\rangle:=\int_{X}\langle g(t), f(t)\rangle d \mu(t)
$$

$$
\langle g, f\rangle\left|\leqslant \int_{X}\right|\langle g(t), f(t)\rangle \mid d \mu(t) \leqslant \int_{X}\|g(t)\|\|f(t)\| d \mu(t) \leqslant q_{M}(f),
$$

where $M$ is a set in $\mathscr{B}$ such that $\|g(\cdot)\| \in M$.

For the implication in one direction, assume that $E^{\prime}$ has the Radon-Nikodým property with respect to $\mu$. First, we deal with the case when $X$ is compact. Let $u$ be a continuous linear form on $\Lambda(E)$. Then there is some $M \in \mathscr{B}$ such that $|u(f)| \leqslant q_{M}(f)$ for all $f \in \Lambda(E)$. For each measurable set $A$, define $G(A)$ by

$$
\langle G(A), x\rangle:=u\left(x \cdot \chi_{A}\right), \quad \text { for } x \in E .
$$


Since $|\langle G(A), x\rangle|=\left|u\left(x \cdot \chi_{A}\right)\right| \leqslant q_{M}\left(x \cdot \chi_{A}\right)=p_{M}\left(\chi_{A}\right) \cdot\|x\|, G$ defines a vector measure on $E^{\prime}$. Clearly $G(A)=0$ whenever $\mu(A)=0$. We prove now that $G$ is $\sigma$-additive. Let $\left(A_{n}\right)$ be a sequence of disjoint measurable sets with union $A$. For each $n \in \mathbb{N}$, the function

$$
\phi_{n}(t)=\chi_{A}(t)-\sum_{i=1}^{n} \chi_{A_{i}}(t)
$$

is in $\Lambda$ because $X$ is compact and $\Phi \subset \Lambda$. Moreover, the sequence $\left(\phi_{n}\right)$ decreases to zero. Now, by hypothesis, $\beta\left(\Lambda, \Lambda^{\times}\right)$equals the Mackey topology $\tau\left(\Lambda, \Lambda^{\times}\right)$and $\phi_{n} \rightarrow 0$ in this topology (see [9], lemma 1). Therefore for all $x \in E$ we have

$$
\left|\left\langle G(A)-\sum_{i=1}^{n} G\left(A_{i}\right), x\right\rangle\right|=\left|u\left(\phi_{n} \cdot x\right)\right| \leqslant q_{M}\left(\phi_{n} . x\right)=p_{M}\left(\phi_{n}\right) .\|x\| .
$$

As $n \rightarrow \infty$, the latter expression converges to zero uniformly on the unit ball of $E$. Hence $G(A)=\sum_{n=1}^{\infty} G\left(A_{n}\right)$ in the dual norm of $E^{\prime}$.

Let us show now that $G$ has bounded variation. Let $\left\{A_{1}, A_{2}, \ldots, A_{n}\right\}$ be a partition of $X$ and take arbitrary points $x_{1}, x_{2}, \ldots, x_{n}$ in the unit ball of $E$. Take scalars $\alpha_{1}, \alpha_{2}, \ldots, \alpha_{n}$ of modulus 1 such that

$$
\left|\left\langle G\left(A_{i}\right), x_{i}\right\rangle\right|=\left\langle G\left(A_{i}\right), \alpha_{i} x_{i}\right\rangle \quad(1 \leqslant i \leqslant n) .
$$

Then every $z_{i}=\alpha_{i} x_{i}(1 \leqslant i \leqslant n)$ is in the unit ball of $E$ and we have

$$
\begin{aligned}
\sum_{i=1}^{n}\left|\left\langle G\left(A_{i}\right), x_{i}\right\rangle\right| & =\left|\sum_{i=1}^{n}\left\langle G\left(A_{i}\right), z_{i}\right\rangle\right|=\left|u\left(\sum_{i=1}^{n} z_{i} \cdot \chi_{A_{i}}\right)\right| \\
& \leqslant q_{M}\left(\sum_{i=1}^{n} z_{i} \cdot \chi_{A_{i}}\right) \leqslant p_{M}\left(\sum_{i=1}^{n} \chi_{A_{i}}\right)=p_{M}\left(\chi_{X}\right) .
\end{aligned}
$$

Taking appropriate suprema shows that $G$ has bounded variation. Since $E^{\prime}$ has the Radon-Nikodým property with respect to $\mu$, there is $g \in L^{1}\left(\mu, E^{\prime}\right)$ such that

$$
G(A)=\int_{A} g d \mu
$$

Let $\left(B_{n}\right)$ be an increasing sequence of measurable sets such that $X=\bigcup_{n=1}^{\infty} B_{n}$ and $g$ is bounded on each $B_{n}$. For each $n \in \mathbb{N}$, consider the functional $u_{n}$ defined by

$$
u_{n}(f)=\int_{B_{n}}\langle g(t), f(t)\rangle d \mu(t) \quad \text { for } f \in \Lambda(E) .
$$

This functional $u_{n}$ is linear. It is also continuous because $g$ is bounded on $B_{n}$ so that $\left\|\chi_{B_{n}}(\cdot) g(\cdot)\right\| \in \Phi \subset \Lambda^{\times}$. Clearly $u_{n}$ agrees with $u$ on all simple functions supported on $B_{n}$. Fix $f \in \Lambda(E)$. Using again [9], lemma 1 to ensure that $f \cdot \chi_{B_{n}} \rightarrow f$ in $\Lambda(E)$, and the fact that simple functions are dense in $\Lambda(E)$, we have

$$
u(f)=\lim _{n} u\left(f \cdot \chi_{B_{n}}\right)=\lim _{n} u_{n}\left(f \cdot \chi_{B_{n}}\right)=\lim _{n} \int_{X}\left\langle g(t), f(t) \cdot \chi_{B_{n}}(t)\right\rangle d \mu(t) .
$$


Since $\Lambda$ is solid, we can apply the equality above to $f . \phi$ for each scalar function $\phi \in L^{\infty}(\mu)$, and this enables us to use the Monotone Convergence Theorem to deduce that the function $|\langle g(\cdot), f(\cdot)\rangle|$ is integrable and, in fact,

$$
u(f)=\int_{X}\langle g(\cdot), f(\cdot)\rangle d \mu(t)
$$

We now consider the $\sigma$-compact case. Write $X=\bigcup_{n=1}^{\infty} X_{n}$ where each $X_{n}$ is compact and $X_{n} \subset$ int $\left(X_{n+1}\right)$. Given $u \in \Lambda(E)^{\prime}$ and $n \in \mathbb{N}$, let $u_{n}$ be the restriction of $u$ to the space $\Lambda_{X_{n}}(E):=\left\{f \cdot \chi_{X_{n}}: f \in \Lambda(E)\right\}$. This is a complemented subspace in $\Lambda(E)$. Then $u_{n} \in \Lambda_{X_{n}}(E)^{\prime}$. Therefore, as proved above, there is a function $g_{n} \in L_{X_{n}}^{1}\left(E^{\prime}\right)$ such that

$$
u_{n}\left(f \cdot \chi_{X_{n}}\right)=\int_{X_{n}}\left\langle g_{n}(t), f(t)\right\rangle d \mu(t), \quad \text { for } f \in \Lambda(E)
$$

Define $g:=\sum_{n=1}^{\infty} g_{n} \cdot \chi_{Y_{n}}$ where $Y_{1}=X_{1}$ and $Y_{n}=X_{n} \backslash X_{n-1}$ for $n \geqslant 2$. This function $g$ is $\mu$-measurable. For $f \in \Lambda(E)$ we have, reasoning as before, that $|\langle g(\cdot), f(\cdot)\rangle|$ is in $L^{1}$ and

$$
u(f)=\int_{X}\langle g(t), f(t)\rangle d \mu(t)
$$

Finally, take an arbitrary function $h \in \Lambda$, and apply the Lemma above to $h . g$ and the positive function $\epsilon(t)=\sum_{n=1}^{\infty} 2^{-n} \chi_{Y_{n}}(t)\left(\mu\left(Y_{n}\right)+1\right)^{-1}$. Then there is a $\mu$-measurable function $y$ from $X$ into the unit ball of $E$ such that

$$
\|h(t) . g(t)\| \leqslant\langle h(t) \cdot g(t), y(t)\rangle+\epsilon(t) \quad \mu \text {-a.e. }
$$

Consequently, we have

$$
\int_{X}|h(t)| \cdot\|g(t)\| d \mu(t) \leqslant \int_{X}\langle g(t), y(t) . h(t)\rangle d \mu(t)+\mu(X)<+\infty .
$$

This proves that $\|g(\cdot)\| \in \Lambda^{\times}$and, therefore, that $g \in \Lambda^{\times}\left(E^{\prime}\right)$.

For the opposite implication, suppose that $\Lambda(E)^{\prime}=\Lambda^{\times}\left(E^{\prime}\right)$. Again we assume, for the first part of the proof, that $X$ is compact. Let $G$ be a $\sigma$-finite vector measure, having bounded variation on $E^{\prime}$ that vanishes on $\mu$-null sets. Its variation $|G|$ is $\sigma$-additive, finite and also vanishes on $\mu$-null sets. By the classical Radon-Nikodým Theorem, there is $h \in L^{1}(\mu)$ such that $h$ is non-negative and such that for each measurable set $A$ we have

$$
|G|(A)=\int_{A} h d \mu
$$

Let $\left(B_{n}\right)$ be a sequence of disjoint measurable sets covering $X$ and such that $h$ is bounded on each $B_{n}$. For each $n \in \mathbb{N}$, define $u_{n}$ as follows :

$$
u_{n}\left(\sum_{i=1}^{k} x_{i} \cdot \chi_{A_{i}}\right)=\sum_{i=1}^{k}\left\langle G\left(A_{i} \cap B_{n}\right), x_{i}\right\rangle
$$


A straightforward computation shows that $u_{n}$ is a continuous linear functional on the space of simple functions. By the Hahn-Banach Theorem, $u_{n}$ has a continuous linear extension to the whole of $\Lambda(E)$. Since the dual of $\Lambda(E)$ is $\Lambda^{\times}\left(E^{\prime}\right)$, for this extension, that we still call $u_{n}$, there is $g_{n} \in \Lambda^{\times}\left(E^{\prime}\right) \subset L^{1}\left(\mu, E^{\prime}\right)$ such that

$$
u_{n}(f)=\int_{X}\left\langle g_{n}(t), f(t)\right\rangle d \mu(t)
$$

for every $f \in \Lambda(E)$; hence for every measurable set $A$, we have

$$
G\left(A \cap B_{n}\right)=\int_{A \cap B_{n}} g_{n} d \mu .
$$

Applying the Lemma above to $h_{n}:=\sum_{i=1}^{n} g_{i} \cdot \chi_{B_{i}}$ and $\epsilon \equiv 1$, we obtain a countably valued $\mu$-measurable function $y$ from $X$ into the unit ball of $E$, and we write $y=\sum_{j=1}^{\infty} x_{j} \cdot \chi_{C_{j}}$, such that

$$
\left\|h_{n}(t)\right\| \leqslant\left\langle h_{n}(t), y(t)\right\rangle+\epsilon(t), \quad \mu \text {-a.e. }
$$

Writing $B=\bigcup_{i=1}^{n} B_{i}$, we have

$$
\begin{aligned}
\sum_{i=1}^{n} \int_{X}\left\|g_{i}(t) \cdot \chi_{B_{i}}(t)\right\| d \mu(t) & =\int_{B}\left\|h_{n}(t)\right\| d \mu(t) \leqslant \sum_{j=1}^{\infty} \int_{B \cap C_{j}}\left\langle h_{n}(t), x_{j}\right\rangle d \mu(t)+\mu(B) \\
& =\sum_{j=1}^{\infty}\left\langle G\left(B \cap C_{j}\right), x_{j}\right\rangle+\mu(B) \leqslant|G|(B)+\mu(B) \\
& \leqslant \int_{X} h(t) d \mu(t)+\mu(X) .
\end{aligned}
$$

Now, define the function $g$ from $X$ to $E^{\prime}$ by

$$
g(t)=\sum_{n=1}^{\infty} g_{n}(t) \cdot \chi_{B_{n}}(t) \in E^{\prime} \quad \text { for all } t \in X
$$

Then $g$ is $\mu$-measurable, and, by applying the Monotone Convergence Theorem to the chain of inequalities above, we have $\|g(\cdot)\| \in L^{1}(\mu)$. Moreover, given a measurable set $A$, we have

$$
G(A)=\sum_{n=1}^{\infty} G\left(A \cap B_{n}\right)=\sum_{n=1}^{\infty} \int_{A \cap B_{n}} g d \mu
$$

and by the Dominated Convergence Theorem

$$
G(A)=\int_{A} g d \mu
$$

Finally, let $X=\bigcup_{n=1}^{\infty} X_{n}$ where each $X_{n}$ is compact and $X_{n} \subset \operatorname{int}\left(X_{n+1}\right)$. Given a $\sigma$-finite vector measure $G$ on $E^{\prime}$ that vanishes on $\mu$-null sets and has bounded 
variation, let $G_{n}$ be its restriction to the corresponding $\sigma$-algebra in $X_{n}$. Since it is clear from our hypothesis that $\left(\Lambda_{X_{n}}(E)\right)^{\prime}=\left(\Lambda_{X_{n}}\right)^{\times}\left(E^{\prime}\right)$, we can apply the case above to obtain $g \in L_{X_{n}}^{1}\left(E^{\prime}\right)$ such that

$$
G_{n}(A)=\int_{A} g_{n} d \mu
$$

Write $Y_{1}=X_{1}$ and $Y_{n}=X_{n} \backslash X_{n-1}$ for $n \geqslant 2$. Define $g:=\Sigma_{n} g_{n} \cdot \chi_{Y_{n}}$. Then $g$ is $\mu$ measurable and

Moreover

$$
G(A)=\sum_{n=1}^{\infty} G_{n}\left(A \cap Y_{n}\right)=\sum_{n=1}^{\infty} \int_{A \cap Y_{n}} g_{n} d \mu=\sum_{n=1}^{\infty} \int_{A \cap Y_{n}} g d \mu .
$$

$$
\int_{X_{n}}\|g(t)\| d \mu(t)=|G|\left(X_{n}\right) \leqslant|G|(X)<+\infty .
$$

Then, by the Monotone Convergence Theorem, $\|g(\cdot)\| \in L^{1}$ and, in fact,

$$
G(A)=\int_{A} g d \mu .
$$

Thus $g \in L^{1}\left(\mu, E^{\prime}\right)$ is the integrable function representing $G$.

Theorem 2. Assume that the dual of $\Lambda\left(\beta\left(\Lambda, \Lambda^{\times}\right)\right)$is $\Lambda^{\times}$. If $E^{\prime}$ has the Radon-Nikodym property with respect to $\mu$ then

(1) a set $H \subset \Lambda(E)^{\prime}=\Lambda^{\times}\left(E^{\prime}\right)$ is equicontinuous if and only if there is $M \in \mathscr{B}$, the family of all normal and $\sigma\left(\Lambda^{\times}, \Lambda\right)$-bounded subsets of $\Lambda^{\times}$, such that $H \subset M$. $U$, where $U$ is the unit ball of $L^{\infty}\left(\mu, E^{\prime}\right)$, and

(2) the strong topology $\beta\left(\Lambda^{\times}\left(E^{\prime}\right), \Lambda(E)\right)$ is the natural topology of the space $\Lambda^{\times}\left(E^{\prime}\right)$ when $\Lambda^{\times}$is endowed with the strong topology $\beta\left(\Lambda^{\times}, \Lambda\right)$ and $E^{\prime}$ carries its dual norm.

Proof. By Theorem 1 we know that $\Lambda(E)^{\prime}=\Lambda^{\times}\left(E^{\prime}\right)$.

(1) Suppose that $H \subset M$.U for some $M \in \mathscr{B}$. Then, of course, $H \subset \Lambda^{\times}\left(E^{\prime}\right)$ and for $f \in \Lambda(E)$ and $g \in H$ we have

$$
\langle g, f\rangle\left|\leqslant \int_{X}\right|\langle g(t), f(t)\rangle \mid d \mu(t) \leqslant \int_{X}\|g(t)\| \cdot\|f(t)\| d \mu(t) \leqslant q_{M}(f) .
$$

Conversely, if $H \subset \Lambda(E)^{\prime}$ is an equicontinuous set, then there is an absolutely convex and closed set $M \in \mathscr{B}$ such that $|\langle g, f\rangle| \leqslant q_{M}(f)$ for all $f \in \Lambda(E)$ and $g \in H$. As we pointed out, every $g \in H$ can be written as $g=\|g\| . h$ where $h \in U$, the unit ball of $L^{\infty}\left(\mu, E^{\prime}\right)$. Therefore it suffices to show that $\{\|g(\cdot)\|: g \in H\} \subset M$. Let $g \in H$, let $\phi$ be in the polar $M^{\circ}$ of $M$ in $\Lambda$, and let $\alpha>0$. By applying the Lemma above to $\phi . g$ and $\epsilon(t)=\sum_{n=1}^{\infty} \alpha 2^{-n} \chi_{Y_{n}}(t)\left(\mu\left(Y_{n}\right)+1\right)^{-1}$, where $Y_{1}=X_{1}$ and $Y_{n}=X_{n} \backslash X_{n-1}$ for $n \geqslant 2$, we obtain a $\mu$-measurable function $y$ from $X$ into the unit ball of $E$ such that

$$
\|\phi(t) \cdot g(t)\| \leqslant\langle\phi(t) \cdot g(t), y(t)\rangle+\epsilon(t), \quad \mu \text {-a.e. }
$$

Now, by integration on $X$

$$
\begin{aligned}
\int_{X}|\phi(t)| \cdot\|g(t)\| d \mu(t) & \leqslant \int_{X}\langle g(t), \phi(t) \cdot y(t)\rangle d \mu(t)+\alpha \\
& \leqslant q_{M}(\phi \cdot y)+\alpha=p_{M}(\phi)+\alpha \leqslant 1+\alpha .
\end{aligned}
$$


Since $\alpha$ was an arbitrary positive number, it follows that $\|g(\cdot)\| \in M^{\circ \circ}=M$.

(2) Let $S$ be $\sigma\left(\Lambda(E), \Lambda^{\times}\left(E^{\prime}\right)\right.$-bounded. Since the dual of $\Lambda(E)$ equals $\Lambda^{\times}\left(E^{\prime}\right)$, we have that $S$ is bounded for the natural topology of $\Lambda(E)$. Then $R=\{\|h(\cdot)\|: h \in S\}$ is $\sigma\left(\Lambda, \Lambda^{\times}\right)$-bounded and for $g \in \Lambda^{\times}\left(E^{\prime}\right)$ we have

$$
\sup \left\{\left|\int_{X}\langle g(t), h(t)\rangle d \mu(t)\right|: h \in S\right\} \leqslant \sup \left\{\int_{X}\|g(t)\| \cdot\|h(t)\| d \mu(t): h \in S\right\}=q_{R}(g) .
$$

This proves that every strong seminorm in $\Lambda^{\times}\left(E^{\prime}\right)$ is continuous for the natural topology.

Conversely, given $R \subset A$ normal and $\sigma\left(\Lambda, \Lambda^{\times}\right)$-bounded, define $S:=R . B$, where $B$ is the unit ball of $L^{\infty}(\mu, E)$. Then $S$ is $\sigma\left(\Lambda(E), \Lambda^{\times}\left(E^{\prime}\right)\right)$-bounded. Now, given $g \in \Lambda^{\times}\left(E^{\prime}\right)$ and $\alpha>0$ there is some $\phi \in R$ such that

$$
q_{R}(g) \leqslant \alpha+\int_{X}|\phi(t)| \cdot\|g(t)\| d \mu(t) .
$$

By application of the Lemma above to $\phi . g$ and $\epsilon(t)=\sum_{n=1}^{\infty} \alpha 2^{-n} \chi_{Y_{n}}(t)\left(\mu\left(Y_{n}\right)+1\right)^{-1}$, where $Y_{1}=X_{1}$ and $Y_{n}=X_{n} \backslash X_{n-1}$ for $n \geqslant 2$, we obtain a $\mu$-measurable function $y$ from $X$ into the unit ball of $E$ such that

$$
\|\phi(t) . g(t)\| \leqslant\langle\phi(t) . g(t), y(t)\rangle+\epsilon(t), \quad \mu \text {-a.e. }
$$

Calling $h=\phi . y \in S$, we obtain

$$
q_{R}(g) \leqslant 2 \alpha+\int_{X}\langle g(t), h(t)\rangle d \mu(t) \leqslant 2 \alpha+\sup \{|\langle g, f\rangle|: f \in S\} .
$$

Since $\alpha$ was arbitrary, it follows that the seminorm $q_{R}$ is dominated by the strong seminorm corresponding to $S$.

\section{Other permanence properties}

In this last section, we study some properties of the spaces $\Lambda(E)$ related to barrelledness. Note that our first result requires weaker hypotheses than the theorems above.

TheOREM 3. (1) If $\Lambda\left(\beta\left(\Lambda, \Lambda^{\times}\right)\right)$is quasi-barrelled, then $\Lambda(E)$ is also quasi-barrelled. Moreover, if $E$ is a Banach space, then $\Lambda(E)$ is barrelled.

(2) If $\Lambda\left(\beta\left(\Lambda, \Lambda^{\times}\right)\right)$is bornological then $\Lambda(E)$ is also bornological.

Proof. (1) Let $N$ be a $\beta\left(\Lambda(E)^{\prime}, \Lambda(E)\right)$-bounded subset of $\Lambda(E)^{\prime}$. We have to prove that $N$ is equicontinuous. For every $u \in N$ and every function $h \in B$, the unit ball of $L^{\infty}(\mu, E)$, consider the composition

$$
T(u, h): \phi \mapsto \phi . h \mapsto\langle u, \phi . h\rangle
$$

from $\Lambda$ to $\mathbb{R}$. It is clear that $T(u, h)$ is a continuous linear form on $\Lambda$. Now, if $R$ is a bounded set in $\Lambda$, then $\{\phi . h: \phi \in R, h \in B\}$ is bounded in $\Lambda(E)$, and since $N$ is strongly bounded, we have

$$
\sup \{|\langle T(u, h), \phi\rangle|=|\langle u, \phi . h\rangle|: u \in N, h \in B, \phi \in R\}<+\infty .
$$


This shows that

$$
N^{*}:=\{T(u, h): u \in N, h \in B\}
$$

is a $\beta\left(\Lambda^{\prime}, \Lambda\right)$-bounded subset of $\Lambda^{\prime}$, the topological dual of $\Lambda\left(\beta\left(\Lambda, \Lambda^{\times}\right)\right)$. Since $\Lambda$ is quasi-barrelled, it follows that $N^{*}$ is equicontinuous. Therefore there is a set $M \in \mathscr{B}$ such that

$$
\sup \{|\langle T(u, h), \phi\rangle|: u \in N, h \in B\} \leqslant p_{M}(\phi) \text {, for all } \phi \in \Lambda \text {. }
$$

Finally, given $f \in \Lambda(E)$ write $f=\|f(\cdot)\| . h$ with $h \in B$. We have

$$
\sup \{|\langle u, f\rangle|: u \in N\} \leqslant \sup \{|\langle T(u, h),\|f(\cdot)\|\rangle|: u \in N, h \in B\} \leqslant q_{M}(f)
$$

and this proves that $N$ is equicontinuous.

If $E$ is a Banach space then $\Lambda(E)$ is complete from [9], proposition 2 and therefore it is a barrelled space from [7], $\$ 27 \cdot 1 \cdot(1)$.

(2) Since $\Lambda$ is bornological, it is quasi-barrelled and, as we have just proved, $\Lambda(E)$ is quasi-barrelled. Now, let $u$ be a locally bounded linear form on $\Lambda(E)$. Using that the bounded sets of $\Lambda(E)$ can be lifted from the bounded sets in $\Lambda$ multiplying by $B$, the unit ball of $L^{\infty}(\mu, E)$, it is easy to see that the set

$$
C:=\{\phi \in \Lambda:|u(\phi . h)| \leqslant 1 \text { for all } h \in B\}
$$

is absolutely convex and bornivorous in $\Lambda$. Since $\Lambda$ is bornological, $C$ is a neighbourhood of zero in $\Lambda$. Hence there is $M \in \mathscr{B}$ such that $M^{\circ} \subset C$. Therefore, for all $h \in B$ and $\phi \in \Lambda$, we have

$$
|u(\phi . h)| \leqslant p_{M}(\phi)
$$

Finally, for $f \in \Lambda(E)$ write $f=\|f(\cdot)\| . h$ with $h \in B$. Then

$$
|u(f)|=|u(\|f(\cdot)\| . h)| \leqslant p_{M}(\|f(\cdot)\| \cdot h)=q_{M}(f),
$$

so that $u$ is continuous. By [7], $\$ 28 \cdot 1 \cdot(3), \Lambda(E)$ is bornological.

For a study of when $\Lambda(E)$ is a barrelled space when $E$ is a normed space, not necessarily complete, we refer the interested reader to [5]. For our next result, recall that a locally convex space is said to be distinguished if its strong dual is a barrelled space.

Theorem 4. Assume that the dual of $\Lambda\left(\beta\left(\Lambda, \Lambda^{\times}\right)\right)$is $\Lambda^{\times}$. If $\Lambda$ is distinguished and $E^{\prime}$ has the Radon-Nikodym property with respect to $\mu$, then $\Lambda(E)$ is distinguished.

Proof. By Theorems 1 and 2, the strong dual of $\Lambda(E)$ is the space $\Lambda^{\times}\left(E^{\prime}\right)$ endowed with its natural topology, when we consider in $\Lambda^{\times}$the strong topology $\beta\left(\Lambda^{\times}, \Lambda\right)$. Since $\Lambda^{\times}\left(\beta\left(\Lambda^{\times}, \Lambda\right)\right)$ is barrelled and $E^{\prime}$ is a Banach space, the space $\Lambda^{\times}\left(E^{\prime}\right)$ is barrelled, by Theorem 3 .

Theorem 5. If $\Lambda$ and $E$ are reflexive then $\Lambda(E)$ is reflexive.

Proof. Assume that $\Lambda$ and $E$ are reflexive. Then, in particular, $\Lambda$ is barrelled and $E^{\prime}$ has the Radon-Nikodým property with respect to $\mu$. By Theorems 1 and 2, the strong dual of $\Lambda(E)$ is the space $\Lambda^{\times}\left(E^{\prime}\right)$ endowed with its natural topology, when $\Lambda^{\times}$ carries the strong topology $\beta\left(\Lambda^{\times} \Lambda\right)$.

Now the dual of $\Lambda^{\times}\left(\beta\left(\Lambda^{\times}, \Lambda\right)\right)$ equals $\Lambda$ because $\Lambda$ is reflexive, and $E^{\prime \prime}=E$ has the Radon-Nikodým property with respect to $\mu$ because it is reflexive. Therefore we can 
apply again Theorems 1 and 2 to $\Lambda^{\times}\left(E^{\prime}\right)$ and deduce that the dual of this space is precisely $\Lambda(E)$. Therefore $\Lambda(E)$ coincides with its bidual and, by Theorem 3 , is barrelled. Hence $\Lambda(E)$ is reflexive.

This research was partially supported by La Consejería de Educación y Ciencia de la Junta de Andalucía.

\section{REFERENCES}

[1] P. Clauzure. Contribution a l'étude topologique et geometrique d'espaces de type Köthe-Orlicz. These, Université des Ciencies et Techniques du Languedoc, Montpellier (1985).

[2] J. Difstel and J. J. Uht, JR. Vector Measures. Mathematical Surveys and Monographs no. 15 (American Mathematical Society, 1977).

[3] J. Dieudonné. Sur les espaces de Köthe. J. Analyse Math. 1 (1951), 81-115.

[4] M. Florencio, P. J. Paúl and C. Sáez. A note on the Köthe dual of Banach-valued echelon spaces. Collect. Math. 39 (1988), 257-261.

[5] L. Drewnowki, M. Florencio and P. J. Paúl. Barrelled function spaces. In Progress in Functional Analysis. Proceedings of the Peñiscola Meeting 1990, on the occasion of the 60th birthday of Professor M. Valdivia (editors K. D. Bierstedt et al.), Mathematical Studies no. 170 (North-Holland, 1992), pp. 191-199.

[6] C. Jordán Lluch and J. R. Torregrosa Sánchez. A characterization of the duals of some echelon Köthe spaces. Collect. Math. 38 (1987), 229-248.

[7] G. Köтне. Topological Vector Spaces, vol. 1 (Springer-Verlag, 1969).

[8] A. L. Macdonald. Vector valued Köthe function spaces. I. Illinois J. Math. 17 (1973), 533-545. II. Illinois J. Math. 17 (1973), 546-557. III. Illinois J. Math. 18 (1974), 136-146.

[9] N. Phuong-Các. Generalized Köthe function spaces. I. Proc. Cambridge Philos. Soc. 65 (1969), 601-611.

[10] R. C. Rosier. Dual spaces of certain vector sequence spaces. Pacific J. Math. 46 (1973), $487-501$. 